
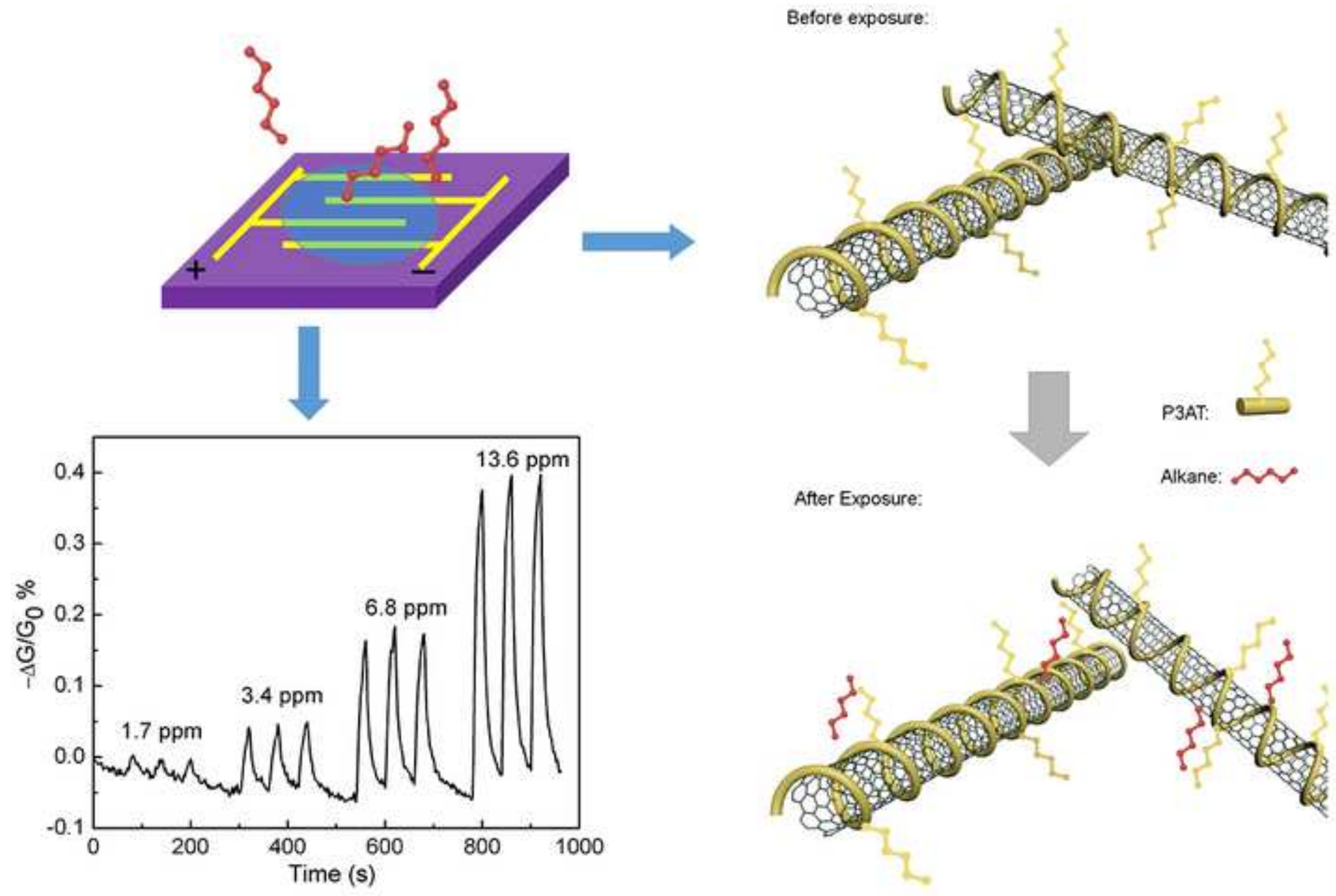

After Exposure:

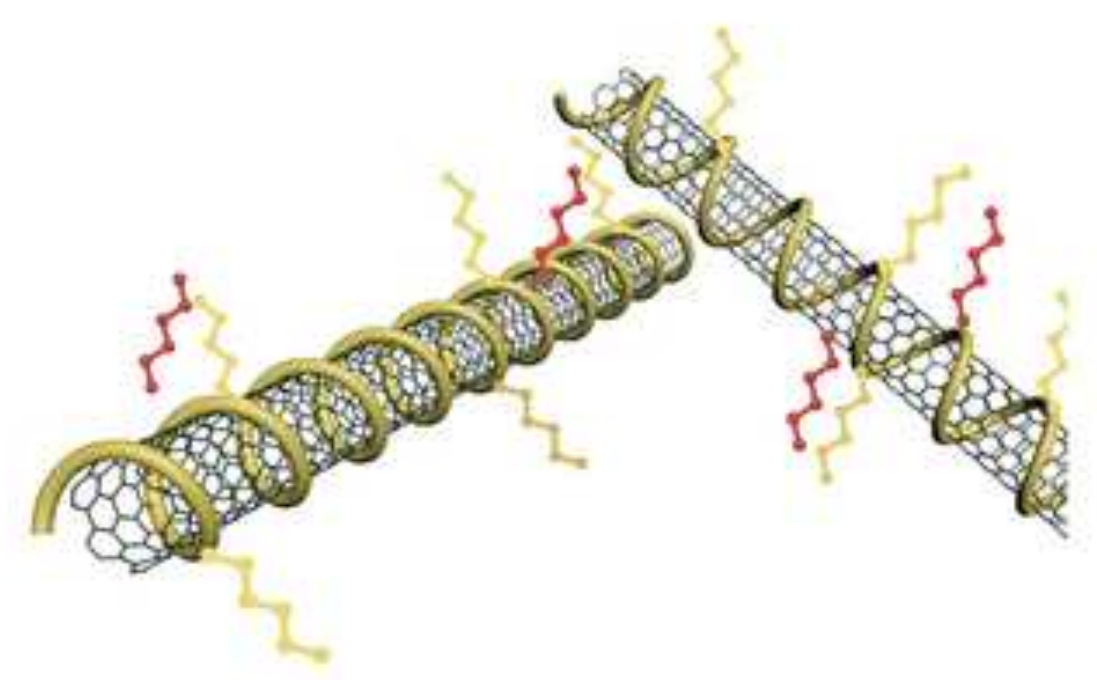




\section{Poly(3-alkylthiophene)/CNT-based Chemiresistive Sensors for Vapor Detection of Linear Alkanes: Effect of Polymer Side Chain Length}

Yaqiong Zhang, ${ }^{a}$ Benjamin R. Bunes, ${ }^{a, b}$ Chen Wang, ${ }^{a} \mathrm{Na} \mathrm{Wu}{ }^{a}$ and Ling Zang ${ }^{a,}{ }^{*}$ 
Abstract: In general, alkane vapors are difficult to detect due to their non-reactive nature at room temperature. Here, we show chemiresistive sensors made of carbon nanotubes (CNTs) noncovalently functionalized with three kinds of poly(3-alkylthiophene) (P3AT), namely, poly(3-butylthiophene) (P3BT), poly(3-octylthiophene-2,5-diyl) (P3OT) and poly(3dodecylthiophene-2,5-diyl) (P3DT). We compared the responses of sensors composed of these materials to four linear alkanes, hexane, octane, decane and dodecane. The results show that sensors with CNTs functionalized with P3ATs that have alkyl side chains with length similar to the length of the analyte alkane produced a bigger response than the case in which the lengths are different. Based on this response trend, a sensor array was made, which can distinguish different sizes of linear alkane vapors. This work facilitates the future design of CNT-based sensor arrays for distinguishing analytes with similar physical and chemical properties.

Keywords: poly(3-alkylthiophene); carbon nanotube; thin film; chemiresistor; vapor sensor

\section{Introduction:}

Alkanes are hydrocarbons with only single carbon-carbon bonds and no functional groups, yet alkanes are important to human beings. They are commonly used as fuels and are also widely used in industry. However, they have safety issues including explosion ${ }^{1}$ and inhalational toxicity. ${ }^{2}$ The Occupational Safety and Health Administration (OSHA) in the United States has regulated the permissible exposure limit of n-hexane to be $500 \mathrm{ppm}^{3}$ Thus, a portable, low-cost, and reliable vapor sensor for detecting and distinguishing alkanes would benefit us in aspects such as detecting fuel leaks in airplanes, detecting homemade explosives made of nitrate-

fuel oil mixtures, and monitoring the environmental exposure of alkanes. However, it is difficult to make highly sensitive alkane sensors due to their lack of reactivity at room temperature and 
they have similar chemical and physical properties. Previous research has been done using traditional instruments, such as Fourier transform infrared (FTIR) spectrometry, ${ }^{4}$ gas chromatography ${ }^{5}$ and ion mobility spectrometry. ${ }^{6}$ However, those methods involve expensive and bulky instruments and the testing processes are time-consuming. Emerging methods have been developed using nanomaterials such as metal oxide thin films, ${ }^{7}$ gold nanoparticles, ${ }^{8-9}$ silicon nanowires, ${ }^{10}$ and organic nanofibers. ${ }^{11}$ However, those sensors usually have specific operational requirements such as high operation temperatures, sophisticated measurement equipment, or light irradiation. Those requirements hinder the development of those sensors.

Chemiresistive sensors based on carbon nanotubes (CNTs) have attracted significant attention due to their high sensitivity, ${ }^{12-13}$ simple fabrication process, ${ }^{14}$ and simple operational requirements. To further enhance their sensitivity and selectivity, covalent ${ }^{15-16}$ and noncovalent ${ }^{17-}$ 18 functionalizations were introduced to CNT-based chemiresistive sensors. Most of these functionalized sensors work through an interaction between the sensor and the analyte such as hydrogen bonding, ${ }^{15}$ halogen bonding, ${ }^{19}$ electrostatic effect, ${ }^{20}$ and the creation of a chemical reaction. ${ }^{21}$ However, none of those methods work in an alkane sensor because of the lack of a specific chemical functional group and the unreactive nature of alkanes at room temperature.

Here, we demonstrate chemiresistive alkane sensors based on CNTs coated with poly(3alkylthiophene) (P3AT). P3ATs with different side alkyl chain lengths were used in this study, namely, poly(3-butylthiophene-2,5-diyl) (P3BT), poly(3-octylthiophene-2,5-diyl) (P3OT), poly(3-dodecylthiophene-2,5-diyl) (P3DT) (Scheme 1a). We have tested the responses of these P3ATs/CNT sensors to four linear alkanes, namely, n-hexane, n-octane, n-decane and ndodecane (Scheme 1b). There are three features in our design. (1) P3ATs greatly enhance the 
dispersion of CNTs in solvents, which makes the fabrication process simple and repeatable. Meanwhile, good dispersion of CNT facilitates the fabrication of a continuous, uniform percolation network of CNTs, which is conducive to gas diffusion and adsorption, thereby facilitating application in vapor sensing. (2) Polythiophenes with a variety of types of side chains are commercially available so we can easily replace the polymer with different side chain lengths and other functional groups to further improve the detection selectivity. (3) The sensors are most responsive to the alkane with similar chain length as the side-chain of P3AT, a mechanism based on the general chemistry principle "like dissolves like". This trend of response could be used to distinguish the size of alkane molecules in a vapor. This research is a proof of concept of designing a sensor array with polymer functionalized CNTs, which can potentially distinguish similar analytes.

\section{Materials and methods:}

\subsection{P3AT/CNT preparation method}

The P3AT/CNT suspensions used for making the sensors were prepared through the following steps. First, CNTs were suspended in 1,2-dichlorobenzene (ODCB) with a concentration of $1 \mathrm{mg} / \mathrm{mL}$, followed by 2 hours of sonication in a sonication bath. P3ATs were dissolved in ODCB with a concentration of $5 \mathrm{mg} / \mathrm{mL}$. Second, the two solutions were mixed to achieve the CNT suspension, in which the weight ratio of P3AT:CNT was 3:1. The mixtures were sonicated for 2 hours in a sonication bath. Subsequently, about 10 vol \% of cyclohexanone was quickly injected into the mixture to facilitate the aggregation of P3ATs onto CNTs. ${ }^{22}$ The mixtures were then kept undisturbed overnight to reach equilibrium. The mixtures thus obtained were relatively homogeneous and dark black. Since the precipitates were not removed, agitation was needed to make the suspension uniform. Third, the mixtures were agitated for several 
seconds and diluted in ODCB to achieve a concentration of CNTs of $2.5 \mu \mathrm{g} / \mathrm{mL}$ (estimated) and the diluted suspensions were sonicated for two hours. Then, the diluted suspensions were ready to use. The diluted suspensions were stable with no aggregates formed over 6 months on a benchtop at room temperature, with the exception of the P3BT/CNT suspension, which formed aggregates after a few weeks (see the photos in Figure 1). After sonicating for several minutes, the aggregation in the diluted suspension of P3BT/CNT would disappear and the suspension would be ready to use again. These uniform and stable P3AT/CNT suspensions make the fabrication process facile and reproducible.

\subsection{Sensor device preparation method}

Sensors were fabricated by drop-casting one of the diluted P3AT/CNT suspensions onto pre-patterned interdigitated electrodes (IDEs) followed by heating at $120{ }^{\circ} \mathrm{C}$ for 10 minutes in an ambient environment to remove the remaining solvent. After the solvent evaporated, the electrical resistance of the IDE was tested. The drop-casting of the suspension was continued until the resistance of the device reached $20-200 \mathrm{k} \Omega$, forming a thin film of P3AT/CNT on the IDEs. Scanning electron microscopy (Figure S2) shows the porous and continuous structure of a $\mathrm{P} 3 \mathrm{OT} / \mathrm{CNT}$ thin film, which is beneficial for a vapor sensor because analyte molecules can penetrate into this thin film.

\section{Results and Discussion:}

\subsection{Characterizations}

UV-Vis absorption spectra were obtained from the three P3AT/CNT suspensions (Figure 1). There were additional peaks around $610 \mathrm{~nm}$ in the P3AT/CNT suspensions, indicating the formation of a highly ordered structure of P3ATs due to their aggregation on the surface of CNTs. ${ }^{23-24}$ The overall baseline of the P3AT/CNT absorption spectra increased when the alkyl 
side chain length increases. This indicates that P3ATs with longer alkyl side chain have stronger abilities to disperse CNTs in solvent. Atomic force microscopy (AFM) was used to characterize the dispersion of CNTs. The AFM image (Figure 1d) indicates that CNTs were individually dispersed. The height distribution study in Figure S-1 shows that the average diameter of the CNTs in the P3OT/CNT suspension is around $1.1 \pm 0.2 \mathrm{~nm}$. Comparing with the manufacture's data and our previous study, ${ }^{25}$ this is an almost $40 \%$ increase of diameter from bare CNTs $(0.8 \pm$ $0.1 \mathrm{~nm}$ ). This demonstrates the addition of the P3OT coating on the surface of CNTs. All those characterizations demonstrate the uniform coating of P3ATs on the surface of the CNTs, as well as the individual dispersion of CNTs thus produced.

\subsection{Sensitivity and limit of detection to n-dodecane}

The sensitivity of those sensors to alkane is first presented with n-dodecane using a P3DT/CNT sensor. Figure 2a shows the three times repeated testing of a P3DT/CNT device by exposing to $1 \%, 2 \%, 4 \%$, and $8 \%$ dilution of saturated vapor of n-dodecane (Table S1 shows the saturated vapor pressures of the four alkane analytes at $20^{\circ} \mathrm{C}$ ). Overall, the sensor's response to $\mathrm{n}$-dodecane is fast, recoverable, and reproducible. Figure $2 \mathrm{~b}$ shows that the sensor displays a linear response to n-dodecane from $1 \%$ to $8 \%$. A limit of detection (LOD) of $342 \mathrm{ppb}$ was projected for n-dodecane following the linear fitting, and similarly a LOD of $76 \mathrm{ppm}$ was projected for $\mathrm{n}$-hexane (see supplementary materials for more detail). The LOD of $76 \mathrm{ppm}$ of $\mathrm{n}$ hexane is far lower than OSHA's permissible exposure limit of $500 \mathrm{ppm}$, demonstrating the utility of the sensor.

\subsection{The effect of side chain length and the sensor array}

Then, the responses of all three P3AT/CNT sensors to all four alkanes were measured. Figure 3 shows the baseline corrected testing results. When exposed to n-hexane (Figure 3a), the 
$\mathrm{P} 3 \mathrm{BT} / \mathrm{CNT}$ sensor gave the biggest response, which is about $45 \%$ larger than the response of P3DT/CNT. However, the P3DT/CNT sensor gave the biggest response to n-dodecane (Figure $3 \mathrm{~b}$ ), which is about $80 \%$ larger than the response of P3BT/CNT. Figure $3 \mathrm{c}$ shows the summary of the sensors' response to all the four alkane analytes. As indicated from the first arrow in Figure 3c, the sensor response to n-hexane decreased as the length of the side chain in the P3AT decreased. On the contrary, the sensor response to n-dodecane increased as the length of the side chain in the P3AT increased. The responses of three kinds of P3AT/CNT sensors to water vapor were tested as well to evaluate the usage in more complex real-life situations (Figure S4). All the sensors show much smaller response to water vapor ( $2504 \mathrm{ppm}, 8 \%$ of saturated vapor) even compared with much lower vapor of dodecane (14 ppm, $8 \%$ of saturated vapor).

The different trends of response observed for the three sensors towards the alkanes vapor as shown in Figure $3 \mathrm{~d}$ demonstrates the capability of distinguishing the different sizes of alkanes by incorporating the three sensors into an array. Figure $3 \mathrm{~d}$ is the principle component score plot of the sensor array. It is clearly seen that the small alkane like n-hexane can be distinguished from the large ones due to the different dissolution preference into the interdigitated polymer side-chain junctions between CNTs. As the size (carbon chain length) of alkanes increases, the dependence of dissolution on the polymer chains becomes less distinct, and consequently it becomes more difficult to discriminate among the long alkanes as shown in Figure 3d.

\subsection{The sensing mechanism}

The mechanism of the sensor is likely due to the swelling of P3ATs on the surface of CNTs. ${ }^{11,26}$ CNTs form a conductive network, while P3ATs act as the insulating layer between each junction of CNTs in the network. Because the electrode gap is much longer than the CNTs, a charge must cross many of these junctions to get from one electrode to the other. When the 
sensors are exposed to alkane vapor, P3ATs on the surface absorb it and swell, causing spatial enlargement of the CNT junctions. As a result, the resistance of the CNT network increased and measured as a decrease in current. The porous structure of the CNT network thin film is easily penetrable by the vapor molecules, and the large interfacial area between adjacent CNTs in the network facilitates the sensor response. Selectivity between alkanes can be understood in two ways. First, shorter alkyl chains of P3AT are less able to accommodate longer alkanes because of their small size; hence, n-hexane elicits a larger response from P3BT/CNT than P3DT/CNT. Conversely, larger alkyl chains of P3AT can accommodate both small and large alkanes, but larger alkanes cause a greater disruption to the interface (i.e., they cause more extensive swelling of the polymer side chains), thus leading to more pronounced sensor response.

\section{Conclusions:}

We utilized P3ATs with three side chain lengths to detect four kinds of alkane vapors at low concentrations. The sensing results indicate that the P3AT/CNT sensors with longer side chain lengths, such as P3DT, show bigger responses to longer carbon chain analytes such as ndodecane. On the contrary, the P3AT/CNT sensors with shorter side chain lengths, such as P3BT, show bigger responses to analytes with shorter carbon chains, such as n-hexane. Thus, by comparing the relative responses of the $\mathrm{P} 3 \mathrm{BT} / \mathrm{CNT}$ sensors and the P3DT/CNT sensors, we were able to selectively detect those alkane analytes within a certain range of vapor concentrations. The sensors were prepared by simply drop-casting P3AT/CNT suspensions onto IDEs. The mechanism of the response is likely due to the swelling of the P3ATs insulting layers in the conductive CNT network. Future work may involve expanding the polythiophene/CNT sensor array with other side chain functional groups, and the sensor array's ability to differentiate a wide variety of analytes and interruptive vapors would be tested. 


\section{Supplementary material:}

Experimental details, height profile of the CNTs from the AFM image, SEM imaging and the calculation of the LOD.

\section{Acknowledgement:}

This work was supported by the Department of Homeland Security, Science and Technology Directorate under Grant (2009-ST-108-LR0005), the National Science Foundation under Grant (IIP-1059286) to the American Society for Engineering Education and USTAR program.

\section{References:}

(1) Eckhoff, R. K. In Explosion Hazards in the Process Industries, 2nd ed; Gulf Professional Publishing: Amsterdam, 2016.

(2) Luttrell, W. E.; Jederberg, W. W.; Still, K. R. Toxicology principles for the industrial hygienist. American Industrial Hygiene Association: Fairfax, VA, 2008.

(3) Occupational Health Guideline for Hexane; U.S. Department of Health and Human Services; U.S. Department of Labor, Washington, DC, 1978

(4) Levitskaia, T. G.; Peterson, J. M.; Campbell, E. L.; Casella, A. J.; Peterman, D. R.; Bryan, S. A., Fourier Transform Infrared Spectroscopy and Multivariate Analysis for Online Monitoring of Dibutyl Phosphate Degradation Product in Tributyl Phosphate/n-Dodecane/Nitric Acid Solvent. Ind. Eng. Chem. Res. 2013, 52, 17607-17617.

(5) Grabowska-Polanowska, B.; Faber, J.; Skowron, M.; Miarka, P.; Pietrzycka, A.; Śliwka, I.; Amann, A., Detection of potential chronic kidney disease markers in breath using gas chromatography with mass-spectral detection coupled with thermal desorption method. $J$. of Chromatogr. A 2013, 1301, 179-189.

(6) M, S.; Vasa, N. J.; Agarwal, V.; Chandapillai, J., UV photo-ionization based asymmetric field differential ion mobility sensor for trace gas detection. Sens. Actuators B Chem. 2014, 195, 44-51. 
(7) Carotta, M. C.; Guidi, V.; Martinelli, G.; Nagliati, M.; Puzzovio, D.; Vecchi, D., Sensing of volatile alkanes by metal-oxide semiconductors. Sens. Actuators B Chem. 2008, 130, 497-501.

(8) García-Berríos, E.; Gao, T.; Woodka, M. D.; Maldonado, S.; Brunschwig, B. S.; Ellsworth, M. W.; Lewis, N. S., Response versus Chain Length of Alkanethiol-Capped Au Nanoparticle Chemiresistive Chemical Vapor Sensors. J. Phys. Chem. C 2010, 114, 21914-21920.

(9) AlQahtani, H.; Sugden, M.; Puzzovio, D.; Hague, L.; Mullin, N.; Richardson, T.; Grell, M., Highly sensitive alkane odour sensors based on functionalised gold nanoparticles. Sens. Actuators B Chem. 2011, 160, 399-404.

(10) Wang, B.; Haick, H., Effect of Chain Length on the Sensing of Volatile Organic Compounds by means of Silicon Nanowires. ACS Appl. Mater.interfaces 2013, 5, 5748-5756.

(11) Wang, C.; Bunes, B. R.; Xu, M.; Wu, N.; Yang, X.; Gross, D. E.; Zang, L., Interfacial Donor-Acceptor Nanofibril Composites for Selective Alkane Vapor Detection. ACS Sensors 2016, $1,552-559$.

(12) Kauffman, D. R.; Star, A., Carbon Nanotube Gas and Vapor Sensors. Angew. Chem. Int. Edit. 2008, 47, 6550-6570.

(13) Li, J.; Lu, Y.; Ye, Q.; Cinke, M.; Han, J.; Meyyappan, M., Carbon Nanotube Sensors for Gas and Organic Vapor Detection. Nano Lett. 2003, 3, 929-933.

(14) Zhang, Y.; Xu, M.; Bunes, B. R.; Wu, N.; Gross, D. E.; Moore, J. S.; Zang, L., Oligomer-

Coated Carbon Nanotube Chemiresistive Sensors for Selective Detection of Nitroaromatic Explosives. ACS Appl. Mater. Interfaces 2015, 7, 7471-7475.

(15) Wang, F.; Swager, T. M., Diverse Chemiresistors Based upon Covalently Modified Multiwalled Carbon Nanotubes. J. Am. Chem. Soc. 2011, 133, 11181-11193.

(16) Ding, M.; Sorescu, D. C.; Star, A., Photoinduced charge transfer and acetone sensitivity of single-walled carbon nanotube-titanium dioxide hybrids. J. Am. Chem. Soc. 2013, 135, 9015-22.

(17) Zhao, Y.-L.; Stoddart, J. F., Noncovalent Functionalization of Single-Walled Carbon Nanotubes. Acc. Chem. Res. 2009, 42, 1161-1171.

(18) Kybert, N. J.; Lerner, M. B.; Yodh, J. S.; Preti, G.; Johnson, A. T. C., Differentiation of Complex Vapor Mixtures Using Versatile DNA-Carbon Nanotube Chemical Sensor Arrays. ACS nano 2013.

(19) Weis, J. G.; Ravnsbæk, J. B.; Mirica, K. A.; Swager, T. M., Employing Halogen Bonding Interactions in Chemiresistive Gas Sensors. ACS Sensors 2016, 1, 115-119. 
(20) Wei, L.; Lu, D.; Wang, J.; Wei, H.; Zhao, J.; Geng, H.; Zhang, Y., Highly sensitive detection of trinitrotoluene in water by chemiresistive sensor based on noncovalently amino functionalized single-walled carbon nanotube. Sens. Actuators B Chem. 2014, 190, 529-534.

(21) Ishihara, S.; Azzarelli, J. M.; Krikorian, M.; Swager, T. M., Ultratrace Detection of Toxic Chemicals: Triggered Disassembly of Supramolecular Nanotube Wrappers. J. Am. Chem. Soc. 2016, 138, 8221-8227.

(22) Ren, S.; Bernardi, M.; Lunt, R. R.; Bulovic, V.; Grossman, J. C.; Gradečak, S., Toward Efficient Carbon Nanotube/P3HT Solar Cells: Active Layer Morphology, Electrical, and Optical Properties. Nano lett. 2011, 11, 5316-5321.

(23) Gu, H.; Swager, T. M., Fabrication of free-standing, conductive, and transparent carbon nanotube films. $A d v$. Mater. 2008, 20, 4433-4437.

(24) Schuettfort, T.; Snaith, H. J.; Nish, A.; Nicholas, R. J., Synthesis and spectroscopic characterization of solution processable highly ordered polythiophene-carbon nanotube nanohybrid structures. Nanotechnology 2010, 21, 025201.

(25) Bunes, B. R.; Xu, M.; Zhang, Y.; Gross, D. E.; Saha, A.; Jacobs, D. L.; Yang, X.; Moore, J. S.; Zang, L., Photodoping and Enhanced Visible Light Absorption in Single-Walled Carbon Nanotubes Functionalized with a Wide Band Gap Oligomer. Adv. Mater. 2015, 27, 162-167.

(26) Severin, E. J.; Doleman, B. J.; Lewis, N. S., An Investigation of the Concentration Dependence and Response to Analyte Mixtures of Carbon Black/Insulating Organic Polymer Composite Vapor Detectors. Anal. Chem. 2000, 72, 658-668.

\section{Figures captions:}

Scheme 1. Molecular structure of the three P3ATs (a) and four linear alkane analytes (b) used in this study.

Figure 1. (a-c) The UV-Vis absorption spectra and photos of P3AT solutions $(0.0075 \mathrm{mg} / \mathrm{ml})$ and P3AT/CNT suspensions. (d) AFM image of the P3OT/CNT suspension cast on a silicon dioxide surface. 
Figure 2. (a) Real-time sensor responses to $1 \%$ (1.7 ppm), 2\% (3.4 ppm), 4\% (6.8 ppm) and 8\% (13.6 ppm) dilution of the saturated vapor of n-dodecane measured from a P3DT/CNT sensor. The analyte exposure time is $20 \mathrm{~s}$ and the recovery time is $40 \mathrm{~s}$. (b) the linear fitting of the sensor response to the vapor concentration of n-dodecane; data points were averaged from three independent sensors' responses.

Figure 3. (a) Real-time sensors' responses to $8 \%$ of the saturated vapor of n-hexane from the P3BT/CNT sensor, the P3OT/CNT sensor and the P3DT/CNT sensor. (b) Real-time sensors' responses to $8 \%$ of the saturated vapor of n-dodecane from the same three sensors. (c) Summary of responses from the three kinds of sensors (three independent sensors for each type) to $8 \%$ of the saturated vapor of n-hexane, n-octane, n-decane, n-dodecane. (d) Principle component score plot of the sensor array containing the three P3AT/CNT sensors to four alkane analytes (three concentrations for each analytes, three trials each concentration). 
(a)

(b)
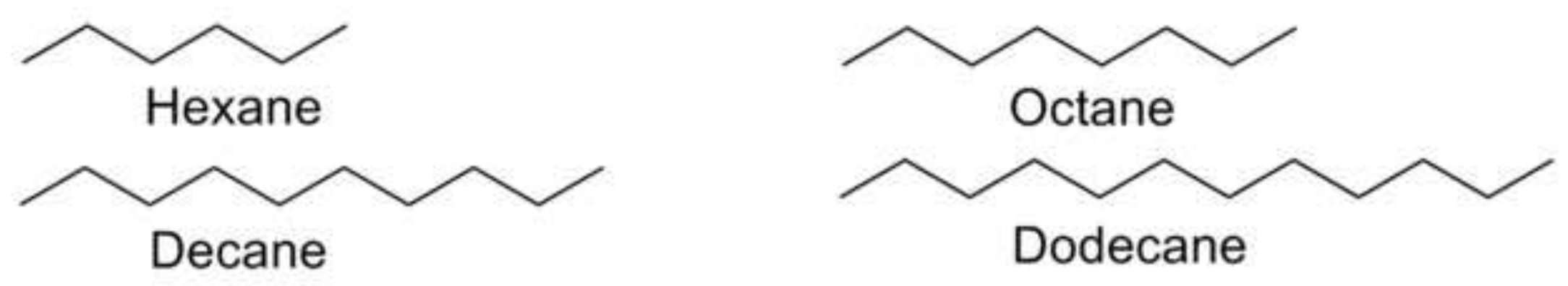

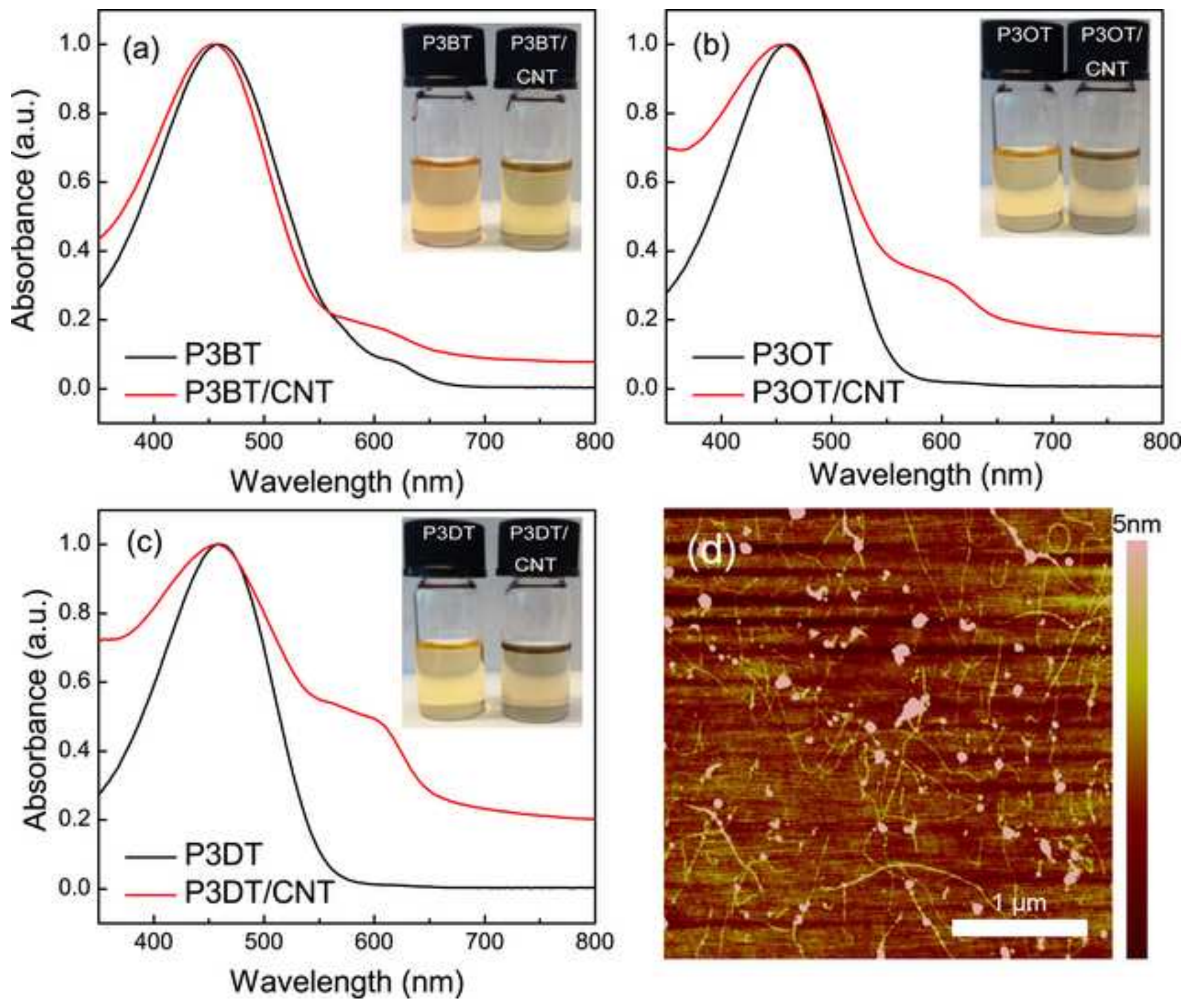

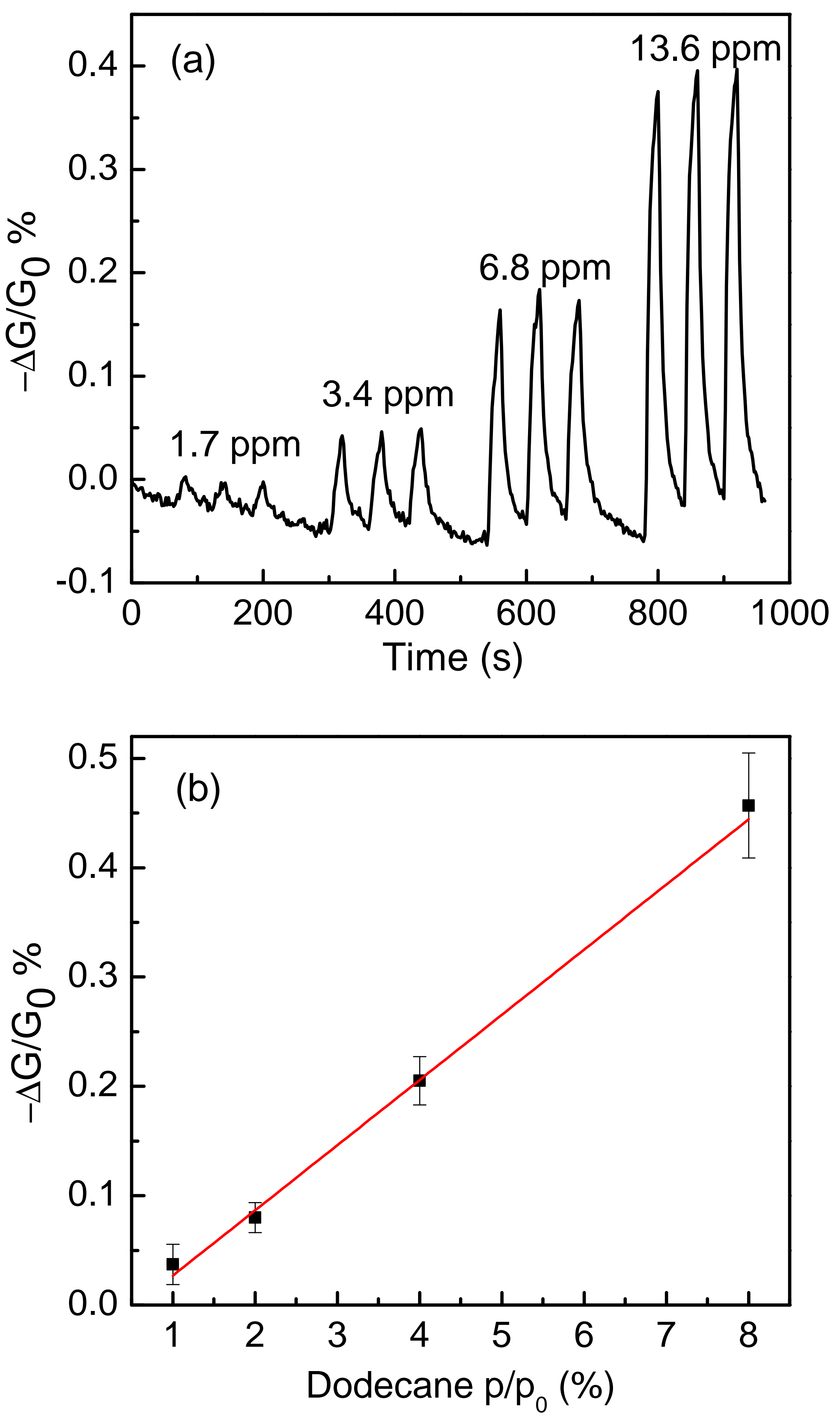
Figure(s)
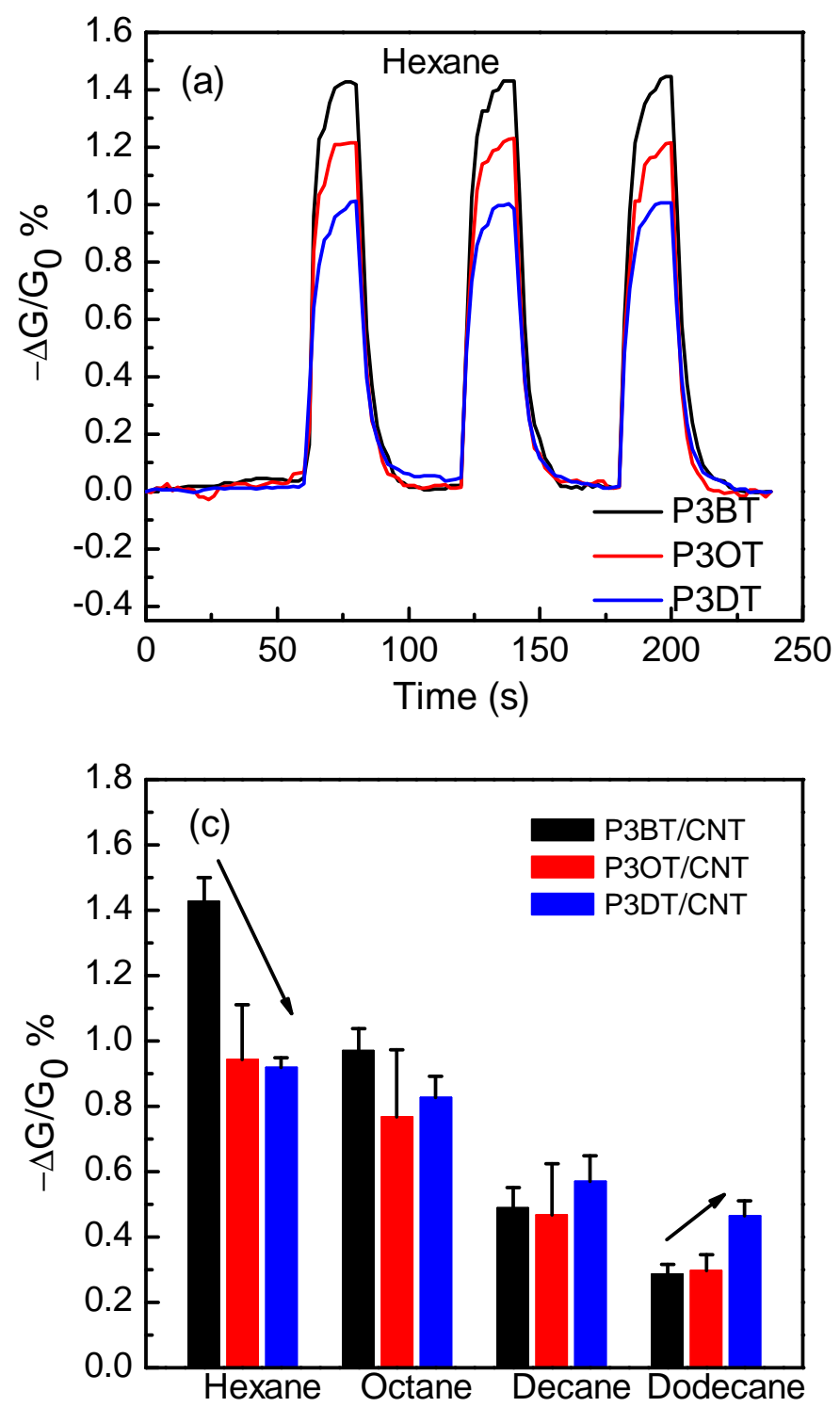
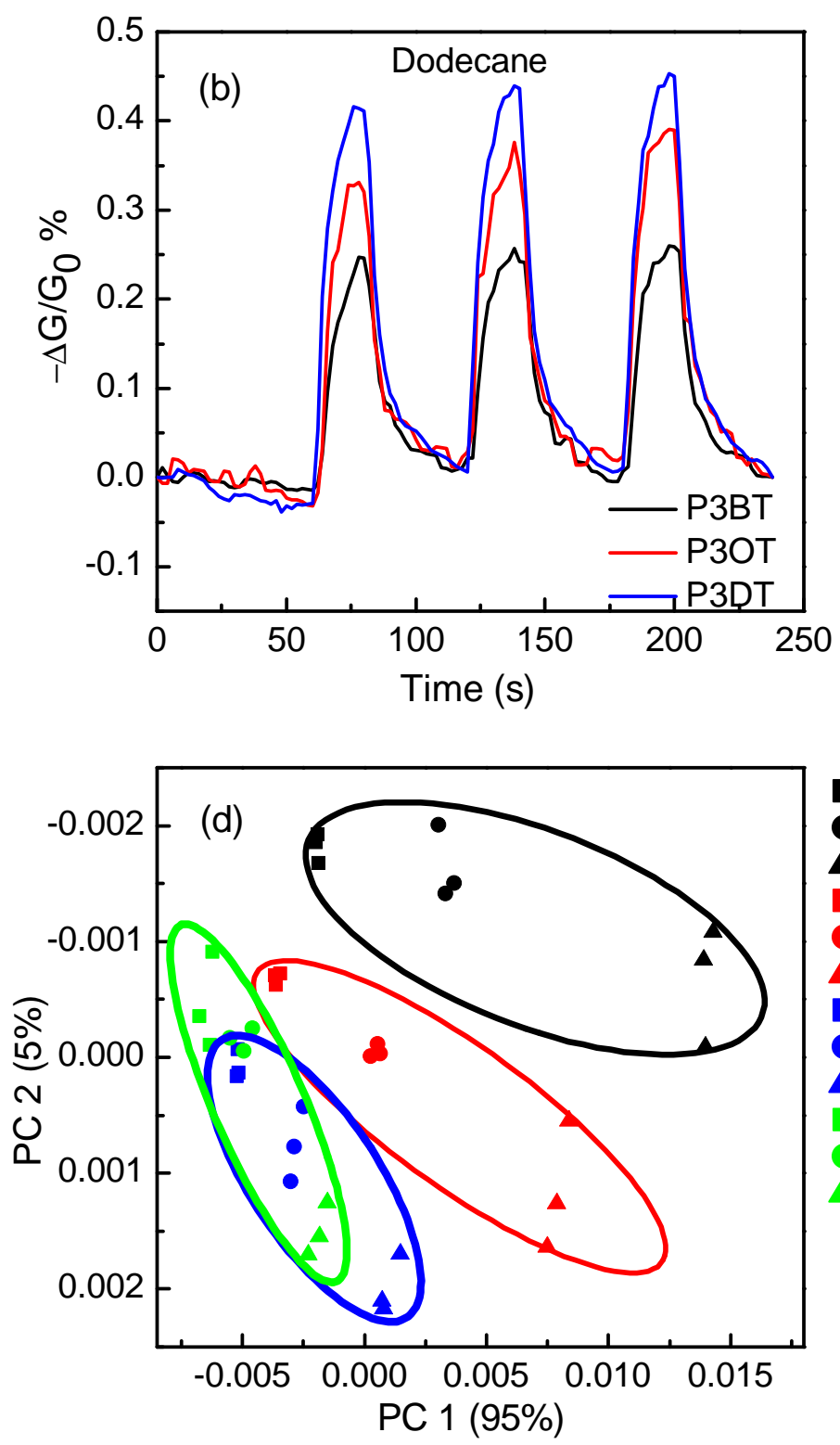

Hexane 2\%

Hexane 4\%

- Hexane $8 \%$

Octane 2\%

Octane 4\%

- Octane $8 \%$

Decane 2\%

- Decane $4 \%$

- Decane $8 \%$

Dodecane 2\%

Dodecane 4\%

Dodecane 8\% 